Draft version OCtober 31, 2018

Preprint typeset using $\mathrm{H}_{\mathrm{T}} \mathrm{EX}$ style emulateapj v. 08/22/09

\title{
THE METALLICITY OF INTERGALACTIC GAS IN COSMIC VOIDS
}

\author{
John T. Stocke, Charles W. Danforth, J. Michael Shull, Steven V. Penton \\ Center for Astrophysics and Space Astronomy, Department of Astrophysical and Planetary Sciences, UCB-389, University of Colorado, \\ Boulder, CO 80309
}

AND Mark L. GiRoux

Department of Physics, Astronomy, \& Geology, East Tennessee State University, Box 70652, Johnson City, TN 37614

Draft version October 31, 2018

\begin{abstract}
We have used the Hubble/STIS and FUSE archives of ultraviolet spectra of bright AGN to identify intergalactic Ly $\alpha$ absorbers in nearby $(z \leq 0.1)$ voids. From a parent sample of 651 Ly $\alpha$ absorbers, we identified 61 "void absorbers" located $>1.4 h_{70}^{-1} \mathrm{Mpc}$ from the nearest $\mathrm{L}^{*}$ or brighter galaxy. Searching for metal absorption in high-quality $(\mathrm{S} / \mathrm{N}>10)$ spectra at the location of three diagnostic metal lines (O VI $\lambda 1032$, C IV $\lambda 1548$, Si III $\lambda 1206$ ), we detected no metal lines in any individual absorber, or in any group of absorbers using pixel co-addition techniques. The best limits on metal-line absorption in voids were set using four strong Ly $\alpha$ absorbers with $N_{\mathrm{HI}}>10^{14} \mathrm{~cm}^{-2}$, with $3 \sigma$ equivalent-width limits ranging from $8 \mathrm{~m} \AA$ (O VI), $7-15 \mathrm{~m} \AA$ (C IV), and 4-10 $\mathrm{m} \AA$ (Si III). Photoionization modeling yields metallicity limits $Z<10^{-1.8 \pm 0.4} Z_{\odot}$ from non-detections of C IV and $\mathrm{O}$ VI, some $\sim 6$ times lower than those seen in Ly $\alpha / O$ VI absorbers at $z<0.1$. Although the void Ly $\alpha$ absorbers could be pristine material, considerably deeper spectra are required to rule out a "universal metallicity floor" produced by bursts of early star formation, with no subsequent star formation in the voids. The most consistent conclusion derived from these low- $z$ results, and similar searches at $z=3-5$, is that galaxy filaments have increased their mean IGM metallicity by factors of $30-100$ since $z \sim 3$.
\end{abstract}

Subject headings: intergalactic medium — quasars: absorption lines — voids — ultraviolet: general - galaxies: dwarfs

\section{INTRODUCTION}

Enormous spatial voids in the large-scale distribution of galaxies were first discovered by Gregory \& Tifft (1976) during a redshift survey of the Coma Cluster of galaxies. Kirshner et al. (1981) found an enormous region of extreme galaxy underdensity in the constellation Boötes, suggesting that voids are a common feature of intergalactic space. The existence and characterization of voids was put on a firm basis by Geller \& Huchra (1989) using the Center for Astrophysics Redshift Survey (CfARS). Since then, every major galaxy survey (e.g., Sloan Digital Sky Survey [SDSS], Las Campanas Redshift Survey [LCRS], H I surveys of Haynes \& Giovanelli [1985]) has discovered new voids, extending our understanding of their size and ubiquity. The SDSS database has also enabled detailed studies of "void galaxies" in the regions of lowest galaxy density. These regions are typically filaments or strings of galaxies that separate immense voids into smaller void volumes. Void galaxies appear to differ in luminosity function (fainter $L^{*}$, Hoyle et al. 2005) and other properties (e.g., higher star formation rate) from similar type galaxies in denser regions (Rojas et al. 2004). On the theoretical side, large volume numerical simulations have been used to study voids (Gottlöber et al. 2003) in a cosmological context, such as estimating their spatial densities and the abun- dance of bound dark-matter halos within them.

In this paper, we describe a new approach to studying voids through the detection of Ly $\alpha$ absorbing clouds within their volumes. This line of research was made possible after the launch of the Hubble Space Telescope (HST) and its UV spectrographs: the Faint Object Spectrograph (FOS), the Goddard High Resolution Spectrograph (GHRS), and the Space Telescope Imaging Spectrograph (STIS). Far-ultraviolet spectra of higher Lyman lines and key metal lines (O VI, C III) were obtained by FUSE, the Far Ultraviolet Spectroscopic Explorer (Moos et al. 2000). With HST, Morris et al. (1991) and Bahcall et al. (1991) discovered low- $z$ Ly $\alpha$ absorption lines in the spectrum of 3C 273. The subsequent HST/FOS Key Project on QSO Absorption Lines (Weymann et al. 1998) found a surprisingly high line frequency, $d \mathcal{N} / d z \approx 35$, of strong Ly $\alpha$ absorbers at low redshifts. The Key Project survey was sensitive to $\mathrm{Ly} \alpha$ absorbers with equivalent widths $W_{\lambda} \geq 240 \mathrm{~m} \AA$, corresponding to column densities $N_{\mathrm{HI}} \geq 10^{14} \mathrm{~cm}^{-2}$ for $b=25 \mathrm{~km} \mathrm{~s}^{-1}$. Higher resolution Ly $\alpha$ studies with GHRS and the STIS/E140M echelle have equivalent-width sensitivities $\left(W_{\lambda} \geq 15 \mathrm{~m} \AA\right.$ or $\left.\log N_{\mathrm{HI}} \geq 12.44\right)$ at $z=0$, comparable to recent Keck Observatory spectra at $z=2-3$. At these sensitivities, Penton, Stocke, \& Shull (2004) observed $d \mathcal{N} / d z \approx 200$ Ly $\alpha$ absorbers per unit redshift.

Our further efforts on low- $z$ IGM absorbers have ex- 
2007) as well as the spatial distribution of galaxies around the Ly $\alpha$ absorbers. Our early studies (Penton, Shull, \& Stocke 2000; Penton, Shull, \& Stocke 2000) demonstrated that, on average, the strong Ly $\alpha$ absorbers trace the galaxy filaments, while most weaker absorbers tend to be found in voids (Penton, Stocke, \& Shull 2002; McLin, et al. 2002). Stocke et al. (1995) targeted bright QSOs and BL Lac Objects behind very nearby voids and discovered a few Ly $\alpha$ absorbers within their boundaries, verifying the presence of baryons in voids. Penton et al. (2004) found that $29 \pm 4 \%$ of cosmological baryons reside in the low- $z$ Ly $\alpha$ forest, while statistical studies (Penton, Stocke, \& Shull 2002) of 81 GHRS-discovered Ly $\alpha$ absorbers showed that $22 \pm 8 \%$ of these Ly $\alpha$ absorbers reside within voids, defined as regions located $>3 h_{70}^{-1} \mathrm{Mpc}$ away from the nearest known bright galaxy, in surveys complete to at least $L^{*}$ galaxy luminosities.

Our most recent survey (Danforth \& Shull 2007) examined the combined FUSE and STIS echelle archives, identifying 651 Ly $\alpha$ absorbers along 28 sight lines. These absorbers and their metal lines can now be compared to existing galaxy redshift surveys, allowing us to identify void absorbers at $z \leq 0.1$. Our earlier work showed that absorbers in voids are preferentially found at lower column densities than absorbers in filaments, with few occurring at $N_{\mathrm{HI}} \geq 10^{14} \mathrm{~cm}^{-2}$. Integrating the absorber numbers (Fig. 12 in Penton, Shull, \& Stocke 2002) using their standard assumptions for cloud sizes $\left(100 h_{70}^{-1} \mathrm{Mpc}\right)$ and ionizing intensities at 1 ryd $\left(J_{0}=10^{-23} \mathrm{erg} \mathrm{cm}^{-2}\right.$ $\left.\mathrm{s}^{-1} \mathrm{~Hz}^{-1} \mathrm{sr}^{-1}\right)$, we estimated that $4.5 \pm 1.5 \%$ of all cosmological baryons are present deep within voids (Penton, Stocke \& Shull 2002; Stocke, Shull, \& Penton 2006). If we assume the standard cosmological (Spergel et al. 2007) mixture of baryons $\left(\Omega_{b}=0.0455 h_{70}^{-2}\right)$ and total matter $\left(\Omega_{m}=0.261 h_{70}^{-2}\right)$ for Hubble constant $H_{0}=$ $\left(70 \mathrm{~km} \mathrm{~s}^{-1} \mathrm{Mpc}^{-1}\right) h_{70}$, the fractional matter density deep within voids is then $\Omega_{\text {void }} \approx 0.015 \pm 0.005$, consistent with numerical simulations of voids in $\Lambda \mathrm{CDM}$ universes (Gottlöber et al. 2003). Thus, the presence of Ly $\alpha$ absorbers within voids appears to be consistent with their formation by gravitational processes.

There are several possibilities for the metal enrichment of Ly $\alpha$ clouds in voids. Because metal-enriched bubbles from galactic winds are unlikely to fill the entire volume of intergalactic space (Ferrara, Pettini, \& Shchekinov 2000; Madau, Ferrara, \& Rees 2001), one might expect some regions to be primordial, with essentially no metals. Alternatively, the IGM may have been seeded with heavy elements from an early era of star formation, such as Pop III stars in dwarf Primordial (dPri) galaxies (Ricotti, Gnedin, \& Shull 2002, 2007). In this case, the dPri galaxies have now faded or lost their gas, and thus would no longer be visible. Some evidence for the latter scenario is suggested by various searches for metals at $z \geq 5$ (Songaila 2001; Pettini et al. 2003) which find evidence for ubiquitous C IV in Ly $\alpha$ absorbers with $N_{\mathrm{HI}} \geq 10^{14} \mathrm{~cm}^{-2}$, at metallicities $Z_{\mathrm{C}} \approx 10^{-3.5} Z_{\odot}$. Co-addition surveys (Cowie \& Songaila 1998 ) of spectra of bright QSOs at $z \approx 2.5-3.0$ detected several heavy elements (C IV, O VI, Si IV) in Ly $\alpha$ absorbers (e.g., Ellison et al. 1999, 2000; Schaye et al. 2003; Aguirre et al. 2004; Simcoe et al. 2004). Some of these surveys conclude that large portions, and perhaps the entire high- $z$ IGM, is metal-enriched to $10^{-2}$ to $10^{-3} Z_{\odot}$ by redshifts $z=2-3$. Schaye et al. (2003) find that $\mathrm{C}$ IV/H I abundances depend on gas overdensity, which is correlated with column density $N_{\mathrm{HI}}$. In their survey at $z \sim 2.5$, Simcoe et al. (2004) do not see a trend of decreasing metallicity toward lower- $N_{\mathrm{HI}}$. Both $[\mathrm{C} / \mathrm{H}]$ and $[\mathrm{O} / \mathrm{H}]$ show log-normal distributions around mean values $\sim 10^{-2.85} Z_{\odot}$, but they find no universal metallicity floor. Some $60-70 \%$ of the absorbers are enriched to levels $Z \geq 10^{-3.5} Z_{\odot}$, but the remaining $\sim 30 \%$ of lines have even lower (or undetectable) metal abundances. Similar conclusions were found by Ellison et al. (1999).

Ultraviolet spectra (HST, FUSE) allow us to test the two hypotheses for IGM metal injection by investigating the "fossil record" of the metallicity of Ly $\alpha$ absorbers in low- $z$ cosmic voids. This test is possible because sites of recent star formation are not seen in their vicinity. For example, McLin et al. (2002) found no galaxies within several hundred kpc of absorbers in very nearby voids $\left(c z \leq 3000 \mathrm{~km} \mathrm{~s}^{-1}\right)$ down to luminosity limits comparable to the luminosities of Local Group dwarf spheroidals. Searches for H I (21-cm) emission near Ly $\alpha$ void absorbers have found no galaxies or galaxy-sized H I clouds (van Gorkom, private communication) to sensitivities of $M_{\mathrm{HI}} \leq 10^{8} M_{\odot}$. These are important constraints, since galactic outflows driven by 10-40 Myr bursts of star formation from high- $z$, low-mass galaxies create fairly small bubbles. Even after $100 \mathrm{Myr}$, the bubble sizes are less than $10-30 \mathrm{kpc}$ for $100-300 \mathrm{~km} \mathrm{~s}^{-1}$ winds. High-speed winds $\left(1000 \mathrm{~km} \mathrm{~s}^{-1}\right)$ from massive galaxies would affect considerably less than $1 \mathrm{Mpc}$ after 1 Gyr.

In $\S 2$, we define our sample of void absorbers, in $\S 3$ we present details of the spectral analysis, and in $\S 4$ we discuss the inferred metallicity limits. In $\S 5$ we present our conclusions and discuss the promise for improving these results after the planned September 2008 installation of the Cosmic Origins Spectrograph (COS) on HST.

\section{THE SAMPLE OF VOID ABSORBERS}

We began our study with the 651 Ly $\alpha$ absorbers at $z \leq 0.4$ and their associated metal lines (O VI, C III, C IV, Si III, Si IV, N V, Fe III) from the survey (Danforth \& Shull 2007) of 28 AGN sight lines observed by both FUSE and STIS echelle (E140M). These data include identifications of the associated O VI (1031.926 $\AA)$, C IV (1548.203 ̊), and Si III (1206.500 ^) absorption. Upper limits can also be obtained for the void absorbers. These three metal lines were judged to provide the best combination of elemental abundance and line strength to serve as probes of absorber metallicity. The far-UV absorption line C III (977.021 $\AA$ ) was not used, as it falls in the $\mathrm{SiC}(\mathrm{low}-\mathrm{S} / \mathrm{N})$ portion of the FUSE band. We also did not explore Si IV (1393.755 ^) in the longer wavelength portion of STIS/E140M. Surveys of Si IV $\lambda 1393.755$ might be useful to accompany C IV $\lambda 1548.2$ as a tracer of the $\mathrm{Si} / \mathrm{C}$ abundance in absorbers with $\log U \approx-1.6 \pm 0.4$. By examining the (Si/C) abundances and the Si IV/C IV line strengths, we estimate that $\mathrm{Si}$ IV/C IV absorption lines should have the ratio $[N f \lambda]_{\mathrm{SiIV}} /[N f \lambda]_{\mathrm{CIV}} \approx 0.32 f_{\mathrm{SiIV}} / f_{\mathrm{CIV}}$, where 
$f_{\mathrm{SiIV}} / f_{\mathrm{CIV}}$ is the ratio of ionization fractions. The $(\mathrm{Si} / \mathrm{C})$ abundance ratio in the IGM might be a factor two higher than the assumed solar ratio, $\mathrm{Si} / \mathrm{C}_{\odot} \approx 0.132$. However, for most values of the ionization parameter, C IV should be a more sensitive probe of metals than Si IV.

To characterize the large-scale distribution of galaxies, we employed our merged galaxy catalog of $>1.3$ million galaxy redshifts (Stocke et al. 2006) from CfA, SDSS/DR6, 2dF, and 6dF surveys, used in our statistical study of O VI absorber environments. In the current paper, we identified voids as regions along the sight line where the three-dimensional "nearest galaxy distance" (NGD) is $>1.4 h_{70}^{-1} \mathrm{Mpc}$ when the galaxy survey was complete to approximately $L^{*}$. We relaxed this criterion from our original NGD minimum distance, from 3.0 to $1.4 h_{70}^{-1} \mathrm{Mpc}$, in order to search for more potential void absorbers. Given the magnitude limits of many current galaxy surveys, this effectively limits our sample to $z_{\text {abs }}<0.1$. To compute the line-of-sight absorber/galaxy distances, we assumed a "retarded Hubble-flow model" (Penton et al. 2002; McLin et al. 2002) in which velocity differences $<300 \mathrm{~km} \mathrm{~s}^{-1}$ are ignored and such objects are placed at the same distance from Earth.

Within our void-absorber sample, we obtained no individual or co-added detections at any value of NGD. Note that our definition of voids is considerably more restrictive than that employed by other investigators (Rojas et al. 2005). However, the number of usable void absorbers remains small (83 at $z<0.1$ ) and varies for different ions, owing to the limited spectral coverage, varying data quality, and occasional confusion with absorption lines of other species at other redshifts (Galactic Ly $\alpha$ and metal lines). We gave further consideration to those absorbers that provide column density limits well below the metal-line detections in the large surveys (Danforth et al. 2006; Danforth \& Shull 2007). This requirement reduced the number of absorbers to 61 , only 12 of which have high-quality data for all three ions of interest. The strongest four H I void absorbers (Table 1) dominate our analysis. Except for the 5040 $\mathrm{km} \mathrm{s}^{-1}$ absorber in the PG 1211+143 sight line (Tumlinson et al. 2005), the strongest low- $z$ void absorbers all lie $>5 h_{70}^{-1} \mathrm{Mpc}$ from the nearest galaxy and are unquestionably deep in voids. Our metallicity limits would not change substantially if we removed from consideration the PG 1211+143 absorber, or the other absorbers with $1.4<\mathrm{NGD}<3 h_{70}^{-1} \mathrm{Mpc}$.

In Table 1 we list by column: (1) the void absorber sight line; (2) heliocentric absorber redshift; (3) NGD in $h_{70}^{-1} \mathrm{Mpc}$; (4) completeness limit of the galaxy survey work around this absorber in $L^{*}$ units; (5) column density $\log N_{\mathrm{HI}}$ of the void absorber; and (6-8) upper limits on metal-ion column densities. Column (9) lists S/N per resolution element at the location of each ion. Dashes in columns 6,7 , and 9 indicate that high-quality data is not available for those ions in that specific absorber. For example, the $z=0.0168$ absorber toward PG 1211+143 has $\mathrm{O}$ VI obscured by Galactic $\mathrm{H}_{2}$ (Tumlinson et al. 2005). A complete table with similar data on all 61 void absorbers in our sample is available upon request.

\section{SPECTRAL ANALYSIS}

In our analysis of the C IV, O VI, and Si III spectra, we considered only those absorbers for which the data are sufficiently good to yield $3 \sigma$ non-detections with $\log N_{\mathrm{CIV}}$ $<12.8, \log N_{\text {OVI }}<13.2$, and $\log N_{\text {SiIII }}<11.8$ in one or both metal lines. These thresholds were determined by examining the distribution of detected column densities and clean (S/N-limited) upper limits in Danforth \& Shull (2007) from which these absorbers were drawn. We made additional cuts from the sample to account for ambiguous void status (e.g., Ton S180 at $c z \sim 13,000 \mathrm{~km} \mathrm{~s}^{-1}$; Stocke et al. 2006 and PKS 0405-123 at $c z \sim 29,000 \mathrm{~km} \mathrm{~s}^{-1}$; Prochaska et al. 2006) and for potential confusion with absorption lines unrelated to the void absorbers. After this culling, the total number of $\operatorname{Ly} \alpha$ absorbers was: O VI (20), C IV (49), and Si III (40). Twelve absorbers, including the 3C 273 entry in Table 1, have clean nondetections in all three ions.

The STIS/E140M and FUSE data were normalized in $10 \AA$ A segments, using low-order Legendre polynomial fits to the line-free continuum regions; see additional discussion in Danforth et al. (2006) and Danforth \& Shull (2007). Absorption features from unrelated IGM systems, ISM lines, and instrumental artifacts were identified interactively and masked for each segment. The signal-to-noise ratio (per resolution element) was found to be close to $S / N=1 / \sigma\left(f_{\text {cont }}\right)$, where $\sigma\left(f_{\text {cont }}\right)$ is the standard deviation in the normalized continuum.

It might seem advantageous to combine data from both lines of the O VI and C IV doublet. However, the benefit from this procedure is, at best, marginal for unsaturated lines. For Poisson noise, in a doublet with 2:1 ratio of oscillator strengths, this procedure increases the $\mathrm{S} / \mathrm{N}$ by a factor $1.5 / \sqrt{2}$, or only $6 \%$. In practice, combining the data often degrades the $\mathrm{S} / \mathrm{N}$ below that of the stronger doublet line alone; sometimes the result is degraded by $20 \%$ or more. We have therefore chosen not to attempt these co-additions. Instead, each ion absorber is represented by a single optical depth vector, $\tau_{\text {ion }}(v)=-\ln \left[I(v) / I_{c}\right]$, which we combine using a pixel-by-pixel, S/N-weighted average of the unmasked pixels. Because the original conversion from flux to optical depth is logarithmic rather than linear, the mean of the combined $\tau$ vectors is slightly greater than zero, $\langle\tau\rangle \sim 0.01$, which we renormalize such that $\langle\tau\rangle=0$. We converted the combined optical-depth vector to a normalized flux, $f(v) / f_{c}(v)=\exp [-\tau(v)]$, and calculated the combined $(\mathrm{S} / \mathrm{N})_{\text {coadd }}=1 / \sigma\left(f_{\text {coadd }}\right)$. Because we see no evidence for significant non-Gaussian errors in either STIS or FUSE data, we characterize the $\mathrm{S} / \mathrm{N}$ by a Gaus$\operatorname{sian} \sigma$.

Since no absorption features were apparent in the combined data, we determined the $3 \sigma$ limits on equivalent width from the total $\mathrm{S} / \mathrm{N}: W_{\max }=\left(3 \lambda_{0} /(R[S / N])\right.$, where the spectral resolution, $R=\lambda / \Delta \lambda$, is taken to be $R_{\text {STIS }} \approx 42,000$ for STIS/E140M (C IV and Si III) and $R_{\mathrm{FUSE}} \approx 15,000$ for FUSE (O VI). Since the optical depth upper limit lies on the linear region of the curve of growth, the column density limit can be calculated from the linear formula, $\left[N_{\text {ion }} / 10^{12} \mathrm{~cm}^{-2}\right]=$ $\left[W_{\lambda} / 8.85 \mathrm{~m} \AA\right]\left[f \lambda_{1000}^{2}\right]^{-1}$, where $f$ is the absorption oscillator strength and $\lambda_{1000}$ is the wavelength in units of $1000 \AA$. For the three metals ions of interest, the parameters (Ion: $f, \lambda_{1000}$ ) have the values: (O VI: 
0.133, 1.032), (C IV: 0.190, 1.548), (Si III: 1.70, 1.206). Thus, in units of $10^{12} N_{12} \mathrm{~cm}^{-2}$, we have $W_{\lambda}$ (C IV) $=(4.03 \mathrm{m \AA}) N_{12}, W_{\lambda}(\mathrm{O}$ VI $)=(1.25 \mathrm{~m} \AA) N_{12}$, and $W_{\lambda}($ Si III $)=(21.9 \mathrm{m \AA}) N_{12}$. We can now compare our void metal-line upper limits with the available $N_{\mathrm{HI}}$ data. Since the spectra were combined using a S/N-weighted average, we perform a weighted average of $N_{\mathrm{HI}}$ to determine a total $N_{\mathrm{HI}}$, but weighted by metal-line $\mathrm{S} / \mathrm{N}$ for each ion. Thus, the final value of $N_{\mathrm{HI}}$ differs for each sample and each ion. In Table 1, we list column densities $N_{\mathrm{HI}}$ and upper limits for $N_{\text {ion }}$ in the four strongest void absorbers.

For 49 void C IV absorbers, we obtain a combined $\mathrm{S} / \mathrm{N}=84.5$ per resolution element, which translates to $W_{3 \sigma}(1548)<1.3 \mathrm{~m} \AA$ and $\log N_{\mathrm{CIV}}<11.51$. Similarly, for $20 \mathrm{O}$ VI void absorbers, we obtain a combined S/N $=48.5$ yielding $W_{3 \sigma}(1032)<4.3 \mathrm{~m} \AA$ and $\log N_{\text {OVI }}$ $<12.53$. Finally, the $40 \mathrm{Si}$ III void absorbers combine to give $\mathrm{S} / \mathrm{N}=84.8$ so that $W_{3 \sigma}(1206)<1.0 \mathrm{~m} \AA$ and $\log N_{\text {SiIII }}<10.67$. The $\mathrm{S} / \mathrm{N}$-weighted mean H I column densities for these three samples are: $\log N_{\mathrm{HI}}=13.46$, 13.47, and 13.55 for the C IV, O VI, and Si III samples, respectively. These translate into mean upper limits for ion ratios of: $N_{\mathrm{CIV}} / N_{\mathrm{HI}}<0.0112, N_{\mathrm{OVI}} / N_{\mathrm{HI}}<0.115$, and $N_{\mathrm{SiIII}} / N_{\mathrm{HI}}<0.0013$. For reference, in our low- $z$ survey of O VI (Danforth \& Shull 2005), the detected $\mathrm{O}$ VI absorbers typically had ion ratios ranging from $N_{\mathrm{OVI}} / N_{\mathrm{HI}} \approx 0.02$ to 2 . This range was interpreted to indicate a wide spread in multiphase ionization conditions of hot and photoionized gas in galaxy filaments.

We also measured integrated equivalent widths across the possible locations of the coadded void metal-line absorption. Most detected metal-line features have FWHM $<50 \mathrm{~km} \mathrm{~s}^{-1}$, so we integrate the coadded $\tau$ and equivalent width $W$ vectors over a range, $v_{\mathrm{HI}} \pm 50 \mathrm{~km} \mathrm{~s}^{-1}$, of ion wavelengths expected from $v_{\mathrm{HI}}$, the $\mathrm{Ly} \alpha$ radial velocity. The standard deviations in the equivalent width measurements correspond to $3 \sigma\left(W_{\text {int }}\right)$ of $0.4 \mathrm{~m} \AA, 1.5 \mathrm{~m} \AA$, and $0.3 \mathrm{~mA}$, for $\mathrm{C}$ IV, O VI, and Si III, respectively, consistent with continuum regions measured in $100 \mathrm{~km} \mathrm{~s}^{-1}$ boxes elsewhere in the coadded data. They are also consistent with the $\mathrm{S} / \mathrm{N}$-derived equivalent width limits above.

Owing to the improved $\mathrm{S} / \mathrm{N}$ of the coadded void absorbers, our coadded ion ratios, $N_{\text {ion }} / N_{\mathrm{HI}}$, are more stringent than those obtained from the individual absorbers in the survey. Table 1 shows that some individual absorbers have even lower ion ratios, owing to a few unusually strong $\mathrm{H}$ I lines $\left(N_{\mathrm{HI}} \geq 10^{14} \mathrm{~cm}^{-2}\right)$. Although the full sample co-addition gives the best indication of the ion ratios for the full ensemble of void absorbers, the most stringent lower limits come from using only the few high column density absorbers in Table 1 . This is explicitly shown in Table 2, which lists ion ratios for several sub-samples of void absorbers. The "Best Individual" absorber is the one whose limits yield the smallest ratio. The "Top Few" sample derives ion ratios from the coaddition of the strongest $\mathrm{H}$ I systems $(1,3$, or 4 for O VI, C IV, and Si III, as listed in Table 1). In this method, we perform a pixel co-addition for the metal lines and adopt a S/N-weighted $N_{\mathrm{HI}}$, assuming that all void absorbers all drawn from the same homogeneous sample with a mean and variance in metallicity. The "Complete Sample" refers to those 12 absorbers with good data in all three ions, and the "All Available" sample uses a pixel coaddition of all 61 void absorbers, although the total used for each ion is somewhat less (20 O VI, $49 \mathrm{C}$ IV and 40 Si III).

Table 2 shows that there are no strong trends in ion ratios for these different sub-samples. We do see small sample-to-sample differences, largely reflecting the mean $N_{\mathrm{HI}}$ and spectral $\mathrm{S} / \mathrm{N}$ of these samples. Therefore, we have chosen to apply the limits on metals obtained from the "Top Few" sample as indicative for the complete sample, since there is no evidence that these differences result from anything more than the $\mathrm{S} / \mathrm{N}$ available in these spectra. Therefore, from Table 2 we choose for further analysis: $N_{\mathrm{CIV}} / N_{\mathrm{HI}}<0.0063, N_{\mathrm{OVI}} / N_{\mathrm{HI}}$ $<0.045$, and $N_{\mathrm{SiIII}} / N_{\mathrm{HI}}<0.00085$. The co-addition method employed here assumes that void absorbers are selected from a uniform population in metallicity. Thus, the upper limits on C IV and Si III from the Top Few sample, derived from pixel co-addition, are $22-26 \%$ lower than from the best individual absorber data.

\section{IONIZATION ANALYSIS}

In order to determine limits on elemental abundances from our limits on individual ion abundances, we must apply ionization corrections. For example, the conversion from a limit on $[\mathrm{OVI} / \mathrm{HI}]$ to a limit on $[\mathrm{O} / \mathrm{H}]$ requires knowledge of the physical state of the gas along the line of sight to the background quasars. Most measurements and models (Danforth \& Shull 2005, 2007; Simcoe et al. 2004) suggest that the IGM absorbers consist of "multiphase gas", in which O VI and other high ions arise in hot, collisionally ionized gas $\left(10^{5-6} \mathrm{~K}\right)$, while $\mathrm{H}$ I and significant portions of C III, Si III, C IV, and Si IV exist in warm $\left(10^{4.0-4.5} \mathrm{~K}\right)$ photoionized gas. Many of these absorbers appear to be kinematically associated within $\pm 20 \mathrm{~km} \mathrm{~s}^{-1}$, but this many not be surprising given the large spatial extents (100-200 kpc) inferred for Ly $\alpha$ absorbers. Because we do not possess detailed knowledge about the cloud geometries or physical structures, we introduce simplified models for the ionization state of the gas, which provide a representative range of metallicity limits.

We begin our analysis with the case of photoionization, which is most appropriate for $\mathrm{C}$ IV and Si III. Collisional ionization will be discussed later. We have constructed several grids of ionization equilibrium models using the photoionization code Cloudy (version 07.02, Ferland et al. 1998). We assume that the gas is optically thin and photoionized by a quasar-dominated metagalactic ionizing background (Haardt \& Madau 1996, 2001), with specific intensity, $J_{\nu}=J_{0}\left(\nu / \nu_{0}\right)^{-\alpha}$. The effective spectral index, $\alpha$, in the Lyman continuum is produced by the range of intrinsic source spectra, altered by transmission and reprocessing through the intervening IGM (Fardal et al. 1998; Shull et al. 1999). Observations of low-redshift AGN (Telfer et al. 2002) suggest intrinsic spectral indices ranging from $0<\alpha_{s}<3$, with a mean $\left\langle\alpha_{s}\right\rangle$ lying somewhere between 1.5 and 2.0. Because transmission tends to harden the radiation spectrum $\left(\alpha<\alpha_{s}\right)$, we scale our results to an effective $\alpha \approx 1.5$ and normalize the ionizing background at 1 ryd 
to $J_{0}=10^{-23} J_{-23}$ erg $\mathrm{cm}^{-2} \mathrm{~s}^{-1} \mathrm{~Hz}^{-1} \mathrm{sr}^{-1}$, close to the derived value at $z \approx 0$ (Shull et al. 1999). When the radiation intensity is fixed, variations in the ionization parameter, $U \propto J_{0} / n_{H}$, represent primarily variations in the hydrogen density $n_{H}$.

To check these effects and their analytic scaling, we examine the following formulae. For a diffuse, isotropic radiation field, $J_{\nu}$, incident on optically thin Ly $\alpha$ clouds from all directions, we can write down formulae for the density, $n_{\gamma}$, of ionizing photons, the density, $n_{H}$, of hydrogen nuclei, the hydrogen photoionization rate, $\Gamma_{H}$, and the photoionization parameter, $U=n_{\gamma} / n_{H}$ :

$$
\begin{aligned}
n_{\gamma} & =\frac{4 \pi J_{0}}{h c \alpha}=\left(4.22 \times 10^{-7} \mathrm{~cm}^{-3}\right) J_{-23}\left(\frac{1.5}{\alpha}\right) \\
n_{H} & =\frac{\rho_{\mathrm{cr}}\left(1-Y_{p}\right) \Omega_{b}}{m_{H}}=\left(1.89 \times 10^{-7} \mathrm{~cm}^{-3}\right) \delta_{H} \\
U & =\frac{n_{\gamma}}{n_{H}}=(2.23) J_{-23}\left(\frac{1.5}{\alpha}\right) \delta_{H}^{-1} \\
\Gamma_{H} & \approx\left[\frac{4 \pi J_{0} \sigma_{0}}{h(\alpha+3)}\right]=\left(2.66 \times 10^{-14} \mathrm{~s}^{-1}\right) J_{-23}\left[\frac{4.5}{\alpha+3}\right]
\end{aligned}
$$

Here, we have written the $(z=0)$ IGM hydrogen density, $n_{H}$, in terms of the baryon mass density $\left(\rho_{\mathrm{cr}} \Omega_{b}\right)$, corrected for primordial helium abundance, $Y_{p} \approx 0.2474$, and scaled to the hydrogen overdensity parameter $\delta_{H}$. The H I photoionization rate involves frequency-integration of $J_{\nu}$ times an approximate photoionization cross section, $\sigma_{\nu} \approx \sigma_{0}\left(\nu / \nu_{0}\right)^{-3}$, where $\sigma_{0}=$ $6.30 \times 10^{-18} \mathrm{~cm}^{2}$ at threshold $h \nu_{0}=1 \mathrm{ryd}$. From their IGM simulations, Davé et al. (1999) found an empirical relation, $\delta_{H} \approx 20 N_{14}^{0.7} 10^{-0.4 z}$, between overdensity $\delta_{H}$ and column density, $N_{\mathrm{HI}}=\left(10^{14} \mathrm{~cm}^{-2}\right) N_{14}$. A similar relation was found by Schaye (2001). We assume photoionization equilibrium, with case-A recombination rate coefficient, $\alpha_{H}^{(A)}=\left(2.5 \times 10^{-13} \mathrm{~cm}^{3} \mathrm{~s}^{-1}\right) T_{4.3}^{-0.76}$, scaled to temperature $T=\left(10^{4.3} \mathrm{~K}\right) T_{4.3}$. We can then derive the photoionization corrections for the $\mathrm{H}$ I neutral fraction, $f_{\mathrm{HI}}$, and estimate the characteristic absorber sizes, $D_{\mathrm{cl}}$ :

$$
\begin{aligned}
& \left.f_{\mathrm{HI}}=\frac{n_{\mathrm{HI}}}{n_{H}}=1.164\left[\frac{n_{H} \alpha_{H}^{(A)}(T)}{\Gamma_{H}}\right] \approx(11.7) n_{H} T_{4.3}^{-0.76} J_{-2}^{-1}(5)\right) \\
& D_{\mathrm{cl}} \approx \frac{N_{\mathrm{HI}}}{n_{H} f_{\mathrm{HI}}} \approx(0.194 \mathrm{Mpc}) N_{14}^{-0.4} T_{4.3}^{0.76} J_{-23}
\end{aligned}
$$

Thus, the observed four strong Ly $\alpha$ absorbers, with $\log N_{\mathrm{HI}}=14.0-14.63$, are predicted to have overdensity factors $\delta_{H}=20-55$ and characteristic sizes $D_{\mathrm{cl}}=$ $100-200 \mathrm{kpc}$. These ranges are probably uncertain to a factor of two. We can conveniently express the photoionization parameter in terms of the parameters, $J_{-23}$ and $\delta_{H}$ :

$$
U=(0.045) J_{-23}\left(\frac{1.5}{\alpha}\right)\left(\frac{50}{\delta_{H}}\right) .
$$

We assume that the absorbers have uniform gas density, $n_{H}$, that the diffuse ionizing radiation, $J_{\nu}$, impinges on the absorbers uniformly from all directions, and that the relative metal abundances are the same as solar photospheric ratios given by Asplund et al. (2005), with $(\mathrm{C} / \mathrm{H})_{\odot}=10^{-3.61},(\mathrm{O} / \mathrm{H})_{\odot}=10^{-3.34}$, and $(\mathrm{Si} / \mathrm{H})_{\odot}=$
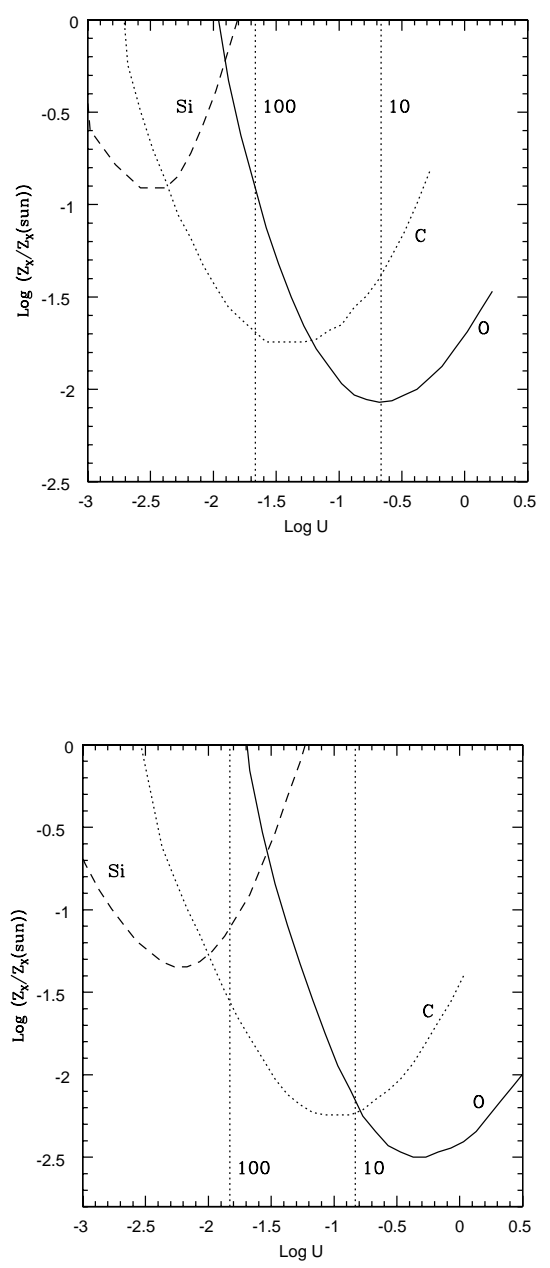

Fig. 1. - Limits on metallicity $\left(Z / Z_{\odot}\right)$ vs. photoionization parameter $(U)$ for silicon, carbon, and oxygen, using observed limits on Si III, C IV, and O VI from Table 1. Ionization corrections were derived from photoionization models of Ferland et al. (1998). $\log U=-1.6 \pm 0.4$ for low- $z$ absorbers (Danforth \& Shull 2007). (Left) Assumes hard ionizing AGN continuum with source spectral index $\alpha_{s}=1.8$ plus IGM transmission. (Right) Assumes mixture of AGN and softer (starburst) continuum. The two vertical dashed lines show overdensities $\left(\delta_{H}=10\right.$ and 100) corresponding to the strong $\operatorname{Ly} \alpha$ absorbers studied here, with fixed $J_{-23}=1$. Metallicity limits, based upon non-detections of C IV and O VI, are $Z<10^{-1.8 \pm 0.4} Z_{\odot}$, assuming $\log U=-1.6 \pm 0.4$ for low- $z$ absorbers (Danforth \& Shull 2007) and including systematic uncertainties of the ionizing spectrum.

$10^{-4.49}$. Previous groups used older abundances (Anders \& Grevesse 1989; Grevesse \& Sauval 1998) in which these abundances were somewhat higher $(\mathrm{C} / \mathrm{H}=331 \mathrm{ppm}$ and $\mathrm{O} / \mathrm{H}=676 \mathrm{ppm}$ ). If we had used these older values, our limits on metal abundances would be 0.17 dex lower for oxygen and 0.13 dex lower for carbon, while the silicon limit would remain unchanged. For a given value of $U$, we vary the global metallicity until we achieve the highest value compatible with the non-detections of Si III, $\mathrm{C}$ IV, and O VI associated with the appropriate $N_{\mathrm{HI}}$.

Figure 1 shows the constraints on [Si,C,O] metallicity 
for a given photoionization parameter associated with upper limits on [Si III, C IV, O VI]. As $U$ increases, the density $n_{H}$ decreases for fixed $J_{0}$, and the upper limit provided by the higher ion stages provides the stronger constraint. The vertical lines in the plot correspond to hydrogen densities, $n_{H}$, assuming an ionizing background $J_{-23}=1$. Our best upper limits on metal absorption lines come from the four specific absorbers, with $N_{\mathrm{HI}}$ ranging from $10^{14.0}$ to $10^{14.63} \mathrm{~cm}^{-2}$, corresponding to $\delta_{H} \approx 20-55$ in the current epoch. For a mean IGM density $\left\langle n_{H}\right\rangle=1.89 \times 10^{-7} \mathrm{~cm}^{-3}$ at $z \approx 0$, the vertical lines in Figure 1 correspond to a somewhat wider range of overdensities $\delta_{H}=10-100$. The best limits on void metallicities come from the absence of $\mathrm{C}$ IV and $\mathrm{O}$ VI, where we adopt a range of photoionization parameter, $\log U \approx-1.6 \pm 0.4$, inferred from the low- $z$ IGM ionization ratios of $\mathrm{Si}$ III/Si IV and C III/C IV found by Danforth \& Shull (2007). The two panels (Fig. 1a,b) show the approximate range of uncertainty in metallicity introduced by the likely range in both $J_{\nu}$ and $\log U$.

The absorbers with lower $N_{\mathrm{HI}}$, listed in Table 2 as the "All Available" sample with $\log \left\langle N_{\mathrm{HI}}\right\rangle=13.5$ (and thus $\log U \approx-1$ ) have metallicity limits set by the absence of $\mathrm{O} \mathrm{VI}$, even for photoionized gas. However, if $J_{0}<10^{-23}$ in voids, or if the radiation field is softer than a HaardtMadau spectrum, the best void metallicity limits are set by $\mathrm{C}$ IV. Unfortunately, at low redshift, there are no strong constraints on $J_{\nu}$ in voids. From Figure 1 we find $Z<10^{-1.8 \pm 0.4} Z_{\odot}$, for $\log U=-1.6 \pm 0.4$. The errors include systematic uncertainties in the assumed ionizing radiation field.

A similar analysis, using $\mathrm{O}$ VI in collisionally-ionized gas, yields metallicity limits $0.5-1.5$ dex lower, depending upon the assumed post-shock temperature of the gas. More likely, O VI is predominantly collisionally ionized, and the $\mathrm{H}$ I resides in photoionized gas. Measurements and models (Danforth \& Shull 2005, 2007; Simcoe et al. 2004) suggest that the IGM absorbers consist of "multiphase gas", in which O VI and other high ions arise in hot, collisionally ionized gas $\left(10^{5-6} \mathrm{~K}\right)$, while H I and significant portions of C III, Si III, C IV, and Si IV exist in warm $\left(10^{4.0-4.5} \mathrm{~K}\right)$ photoionized gas. Many of these absorbers appear to be kinematically associated within $\pm 20 \mathrm{~km} \mathrm{~s}^{-1}$, but this many not be surprising given the large spatial extents $(100-200 \mathrm{kpc})$ inferred for $\operatorname{Ly} \alpha$ absorbers. Numerical simulations of low- $z$ Ly $\alpha$ absorbers find that absorbers far from galaxies are dominated by photoionization (Davé et al. 1999), with collisionally-ionized absorbers found much closer to galaxies. In these models, over $80 \%$ of the absorbers more than $1 \mathrm{Mpc}$ from any galaxy are photo-ionized. Thus, the case of photoionization places the least restrictive and most appropriate limits on metals in voids.

\section{CONCLUSIONS AND FUTURE PROSPECTS}

We have used a large database (Danforth \& Shull 2007) of 651 Ly $\alpha$ absorbers found in both STIS/E140M and FUSE spectroscopy to investigate the metallicity of gas in cosmic voids. We cross-correlated a merged galaxy catalogue with $>1.2$ million entries with the Ly $\alpha$ absorber locations to identify a sample of 61 "void absorbers" at $z<0.1$, located $>1.4 h_{70}^{-1} \mathrm{kpc}$ from the nearest $\mathrm{L}^{*}$ or brighter galaxy. Using both individual spectra of high column density $\left(N_{\mathrm{HI}}>10^{14} \mathrm{~cm}^{-2}\right)$ absorbers and pixeladditions, we find equivalent width limits (O VI, C IV, Si III) of 1-4 mA. The best metallicity limits are set from the strongest 4 absorbers in voids, all of which have $N_{\mathrm{HI}}>10^{14} \mathrm{~cm}^{-2}$ (see Tables 1 and 2). With ionization corrections from standard photoionization models, the metallicity limits $(3 \sigma)$ in voids are $Z<10^{-1.8 \pm 0.2} Z_{\odot}$ for a range of ionization parameters $(\log U=-1.6 \pm 0.4)$ that characterize the low- $N_{\mathrm{HI}}$ IGM at $z<0.1$ (Danforth \& Shull 2007).

Collisionally ionized gas models set even lower metallicity limits, based on the non-detection of O VI. While the results from this first study indicate that cosmic voids contain gas that is quite metal poor, the limit of $Z<10^{-1.8} Z_{\odot}$ by itself is insufficient to constrain the existence of an early burst of star formation within voids. Extremely metal poor (EMP) stars in our own Galaxy have $[\mathrm{Fe} / \mathrm{H}] \leq-3.0$ (Cayrel et al. 2004) in Fe-peak elements, which in some sense sets an upper limit on the metallicity of the earliest stars. However, many of these EMP stars have enhanced $[\mathrm{C} / \mathrm{Fe}]$ and $[\mathrm{O} / \mathrm{Fe}]$ (Christlieb et al. 2002), so the actual metallicities may exceed $1 \%$ solar. Simulations by Ricotti, Gnedin, \& Shull (2002) suggest that IGM metal abundances in the IGM can rise to $\sim 10^{-3.5} Z_{\odot}$ by $z \sim 10$. Assuming standard metal yields, a co-moving star-formation-rate (SFR) density of $\rho_{\mathrm{SFR}} \approx 0.1 M_{\odot} \mathrm{yr}^{-1} \mathrm{Mpc}^{-3}$, typical of the peak values at $z \approx 6$, will produce $1 \%$ solar metallicity in $\sim 1$ Gyr (Tumlinson et al. 2004). The simulations also find an exponentially increasing co-moving rate of star formation from $z=20$ to $z=10$. The early burst of metal production is likely to occur shortly after $z \approx 10$, when simulations suggest that $\rho_{\mathrm{SFR}}$ peaks at $0.1 M_{\odot} \mathrm{yr}^{-1} \mathrm{Mpc}^{-3}$. One scenario is that a few generations of stars formed from low-mass (dPri) galaxies within voids, and then star formation ceased from $z \sim 10$ until the current epoch. In this case, the level of metallicity, $Z \approx 10^{-5} Z_{\odot}$ (Ricotti, Gnedin, \& Shull 2007), would be well below the detection threshold of the current best limits at both high and low redshift.

On the other hand, the mean metallicity of Ly $\alpha$ absorbers in galaxy filaments today has been estimated at $Z \approx 0.1 Z_{\odot}$ (Danforth \& Shull 2005; Stocke et al. 2006), nearly an order of magnitude larger than the $10^{-1.8} Z_{\odot}$ limit derive for voids at $z \leq 0.1$. At much higher redshift, many groups have made detections or set upper limits on metals in the Ly $\alpha$ forest. Songaila (2001) found the $\left(\mathrm{C}\right.$ IV) metallicity at $z=5$ to be $Z \geq 10^{-3.5} Z_{\odot}$, while at $z=2.5-2.8$, Simcoe et al. (2004) found a mean metallicity of oxygen and carbon of $\sim 10^{-2.8 \pm 0.8} Z_{\odot}$, with $\sim 30 \%$ of the absorbers at $N_{\mathrm{HI}} \geq 10^{13.5} \mathrm{~cm}^{-2}$ showing no detectable metals at all, $Z<10^{-3.5} Z_{\odot}$. Ellison et al. (1999, 2000) made similar explorations of weak C IV absorbers down to $\log N_{\mathrm{CIV}} \approx 11.7$ and found no evidence for flattening of the $N_{\text {CIV }}^{-1.44}$ power-law distribution. Pettini et al. (2003) measured 16 C IV systems with $\log N_{\mathrm{CIV}}=12.50-13.98$ at $z>5$, and deduced a comoving mass density $\Omega_{\mathrm{CIV}}=(4.3 \pm 2.5) \times 10^{-8}$ for systems with $\log N_{\mathrm{CIV}}>13.0$. This metal abundance is consistent with that measured at $z<4$ and with the finding (Songaila 2001) that the C IV column-density distribu- 
tion and inferred metallicity show little evolution over this period.

Because void absorbers account for $\sim 22 \%$ of all lowcolumn density Ly $\alpha$ absorbers in the current epoch (Penton, Stocke \& Shull 2002), it is tempting to identify the $30 \%$ metal-free absorbers (Simcoe et al. 2004) at $z \sim 2.5$ with absorbers in voids. Simcoe et al. (2004) use the Grevesse \& Sauval (1998) solar reference abundances, so our best limit is $Z \leq 10^{-1.9} Z_{\odot}$ from $\mathrm{C} / \mathrm{H}$ in Figure 1. If the local void absorbers were drawn from the metallicity distribution at $z \sim 2.5$ quoted above, then we should have detected some metal lines in our current voids sample. From the current evidence, it is easier to identify the metal-bearing population at $z \sim 2.5$ with the $10^{-1} Z_{\odot}$ absorbers in galaxy filaments today, so we suggest that IGM metallicity in filaments has increased by a factor of 30-100 over that time. High-redshift absorbers without metals $\left(Z \leq 10^{-3.5} Z_{\odot}\right)$ probably reside in voids and have remained extremely metal poor or pristine even to the current epoch. If this association of metal-poor absorbers and voids is correct, then the absorbers with $Z \leq 10^{-3.5} Z_{\odot}$ should have a systematically lower twopoint correlation function amplitude than the metal-rich absorbers at comparable redshift.

Our comparisons between high- $z$ and low- $z$ absorbers were made for systems with similar $N_{\mathrm{HI}}$. However, simulations tell us that individual $\operatorname{Ly} \alpha$ absorbers evolve in $N_{\text {HI }}$ over time. Davé et al. (1999) and Schaye et al. (2001) suggest a factor of $\sim 20$ evolution in absorber numbers, from $z \sim 3$ to the present; Penton, Stocke \& Shull (2004) measure a factor of 3-10 over the same range. Therefore, the $z \sim 2.5$ absorbers at $\log N_{\mathrm{HI}}=13.5-15.5$ should become roughly an order of magnitude lower in $N_{\mathrm{HI}}$ in the present epoch. There appears to be some disagreement over the presence of metallicity-density correlation in fits to $[\mathrm{C} / \mathrm{H}]$ or $[\mathrm{O} / \mathrm{H}]$ with $\log N_{\mathrm{HI}}$. Schaye et al. (2003) find a density dependence in their C IV survey, but Simcoe et al. (2004) suggest that these effects may be masked by systematic uncertainties in the ionization radiation field. The interpretation of these trends is tied to uncertainties in the sources and spectral shapes of the UVX background. (Giroux \& Shull 1997; Shull et al. 1999). With no apparent dependence of metallicity with $N_{\mathrm{HI}}$ (Simcoe et al. 2004), our metallicity comparisons remain approximately correct.

While these conclusions are supported by the upper limits derived here for current-epoch void absorbers, they would be strengthened considerably by upper limits $\sim 1$ dex lower. With the Cosmic Origins Spectrograph (COS) awaiting installation into HST, it is worthwhile to consider how these limits could be improved in the COS era.
It will be hard to improve on the oxygen limit, as it is set by the non-detections of O VI by FUSE. At $z \geq 0.12$, the O VI lines shift into the HST/COS band, but current galaxy survey work is of insufficient depth to determine the locations of voids unambiguously at $z \geq 0.1$. Since the current metallicity limit is based largely upon just one $\mathrm{O}$ VI absorber, finding $\sim 10$ new candidate void absorbers at $N_{\mathrm{HI}} \geq 10^{14} \mathrm{~cm}^{-2}$ and $z \geq 0.12$ seems possible, and it could reduce the oxygen abundance limit significantly. Conducting the necessary deep galaxy surveys around a few of these sight lines also seems tractable.

For C IV to set more stringent metallicity limits than $\mathrm{O}$ VI across the full range of $\log U$ would require $\sim 30$ void absorbers with $N_{\mathrm{HI}} \geq 10^{14} \mathrm{~cm}^{-2}$ selected from a large survey of low- $z$ sight lines. Let us assume an absorber frequency, $d N / d z \approx 25$ (Penton et al. 2004) for Ly $\alpha$ absorbers with $N_{\mathrm{HI}} \geq 10^{14} \mathrm{~cm}^{-2}$, with a redshift pathlength $\Delta z \approx 0.1$ per target and a $10 \%$ probability of finding strong $\mathrm{H}$ I absorbers within voids. Therefore, finding 30 strong void absorbers would require observing $\sim 100$ targets. We could reduce this number somewhat by taking longer exposures (higher-S/N spectra) sensitive to weaker metal lines in absorption systems with $\log N_{\mathrm{HI}} \geq 13.6$. An HST/COS survey would rely on current and ongoing galaxy surveys to find the voids. Such target lists exceed the number of AGN now envisioned in the COS Guaranteed Time Observer (GTO) program, but they could be accommodated in a COS Key Project on the IGM. It would marvelous to find just one void absorber with $N_{\mathrm{HI}} \approx 10^{15} \mathrm{~cm}^{-2}$, for which it might be possible to detect metals down to metallicities of $10^{-2.5} Z_{\odot}$ in these strong ultraviolet resonance lines. Given the sensitivity of the O VI lines, a far-UV mission replacing FUSE would be especially important, in order to search for weak, low-redshift $\mathrm{O}$ VI and C III absorbers. The low- $z$ fossil record of metallicity may provide the best evidence for dwarf Primordial galaxies and the first stars (Ricotti et al. 2007).

We acknowledge the financial support from NASA/HST guest observer grants HST-GO-06593.01, HST-GO-09506.01 and from the COS GTO Science Team. Additional support for IGM studies at the University of Colorado came from FUSE (NASA grant NNG06GI91G) and NASA Astrophysical Theory grant NNX07AG77G. JTS thanks Michael Vogeley and Fiona Hoyle for their invitation to speak on this subject at conferences in Aspen and Amsterdam.

\section{REFERENCES}

Aguirre, A., Schaye, J., Kim, T.-S., Theuns, T., Rauch, M., \& Sargent, W. L. W. 2004, ApJ, 602, 38

Anders, E., \& Grevesse, N. 1989, Geochim. Cosmochim. Acta, 53, 197

Asplund, M., Grevesse, N., \& Sauval, A. J., 2005, in ASP Conf. Ser. 336, Cosmic Abundances as Records of Stellar Evolution and Nucleosynthesis, ed. T. G. Barnes III \& F. N. Bash (San Francisco: ASP), 25

Bahcall, J.N. et al. 1991, ApJ, 377, L5

Cayrel, R., et al. 2004, Astr. \& Astr., 416, 1117

Christlieb, N., et al. 2002, Nature, 419, 904

Cowie, L. L., \& Songaila, A. 1998, Nature, 394, 44

Danforth, C. W., \& Shull, J. M. 2005, ApJ, 624, 555

Danforth, C. W., \& Shull, J. M. 2007, ApJ, submitted
Danforth, C. W., Shull, J. M., Rosenberg, J. L., \& Stocke, J. T. 2006, ApJ, 640, 716

Davé, R., Hernquist, L., Katz, J., \& Weinberg, D. 1999, ApJ, 511, 521

Ellison, S. L., Lewis, G. L., Pettini, M. Chaffee, F. H., \& Irwin, M. J. 1999, ApJ, 520, 456

Ellison, S. L., Songaila, A., Schaye, J., \& Pettini, M. 2000, AJ, 120,1175

Fardal, M. A., Giroux, M. L., \& Shull, J. M. 1998, AJ, 115, 2206

Ferland, G. J., Korista, K. T., Verner, D. A., Ferguson, J. W., Kindon, J. B., \& Verner, E. M. 1998, PASP, 110, 761

Ferrara, A., Pettini, M., \& Shchekinov, Yu. 2000, MNRAS, 319, 539

Geller, M. J., \& Huchra, J. H. 1989, Science, 246, 897 
TABLE 1

Four Strong Void Absorbers

\begin{tabular}{lcccccccc}
\hline \hline Sight Line & $z_{\text {abs }}$ & $\begin{array}{c}\mathrm{NGD}^{\mathrm{a}} \\
h_{70}^{-1} \mathrm{Mpc}\end{array}$ & $\begin{array}{c}\text { Limiting }{ }^{\mathrm{b}} \\
L\left(L^{*}\right)\end{array}$ & $\begin{array}{c}\log N_{\mathrm{HI}} \\
\left(\mathrm{cm}^{-2}\right)\end{array}$ & $\begin{array}{c}\log N_{\mathrm{OVI}}{ }^{\mathrm{c}} \\
\left(\mathrm{cm}^{-2}\right)\end{array}$ & $\begin{array}{c}\log N_{\mathrm{CIV}}{ }^{\mathrm{c}} \\
\left(\mathrm{cm}^{-2}\right)\end{array}$ & $\begin{array}{c}\log N_{\mathrm{SiIII}}{ }^{\mathrm{c}} \\
\left(\mathrm{cm}^{-2}\right)\end{array}$ & $\begin{array}{c}(\mathrm{S} / \mathrm{N}) \text { per resel } \\
1032 / 1548 / 1206\end{array}$ \\
\hline 3C 273 & 0.06656 & 6.7 & 1 & $14.17 \pm 0.11$ & $<12.82$ & $<12.27$ & $<11.20$ & $20 / 20 / 35$ \\
3C 351 & 0.04086 & 10.0 & 0.3 & $14.63 \pm 0.14$ & $\cdots$ & $<12.56$ & $<11.67$ & $\cdots / 10 / 12$ \\
PG 1211+143 & 0.01680 & 1.8 & 0.5 & $14.00 \pm 0.15$ & $\cdots$ & $<12.24$ & $<11.46$ & $\cdots / 21 / 19$ \\
PKS 0312-770 & 0.07368 & 11.3 & 0.5 & $14.48 \pm 0.85$ & $\cdots$ & $\cdots$ & $<11.71$ & $\cdots / \cdots / 11$ \\
\hline
\end{tabular}

a NGD $=$ Nearest Galaxy Distance (see text in § 2)

b Nearby galaxy survey completeness limit, in units of $L^{*}$, at absorber redshift

${ }^{\text {c }}$ Upper limits $(3 \sigma)$ of metal ion column densities.

TABLE 2

Metal Ion Limits in Void Absorbers

\begin{tabular}{lccccc}
\hline \hline \multicolumn{1}{c}{ Sample } & $\begin{array}{c}\text { Sample } \\
\text { Size }\end{array}$ & $\begin{array}{c}\text { S/N-weighted } \\
\log \left\langle N_{H I}\right\rangle\end{array}$ & $N_{\mathrm{OVI}} / N_{\mathrm{HI}}$ & $N_{\mathrm{CIV}} / N_{\mathrm{HI}}$ & $N_{\mathrm{SiIII}} / N_{\mathrm{HI}}$ \\
\hline Best Individual & $1 / 1 / 1$ & $14.17 / 14.63 / 14.17$ & $<0.045$ & $<0.0085$ & $<0.0011$ \\
Top Few (Table 1) & $1 / 3 / 4$ & $14.17 / 14.27 / 14.29$ & $<0.045$ & $<0.0063$ & $<0.00085$ \\
Complete Sample & 12 & $13.59 / 13.49 / 13.57$ & $<0.043$ & $<0.019$ & $<0.0025$ \\
All Available & $20 / 49 / 40$ & $13.46 / 13.47 / 13.55$ & $<0.115$ & $<0.0112$ & $<0.0013$ \\
& & & & & \\
\hline
\end{tabular}

Giroux, M. L., \& Shull, J. M. 1997, AJ, 113, 1505

Gottlöber, S., Lokas, E. L., Klypin, A., \& Hoffman, Y. 2003, MNRAS, 344, 715

Gregory, S. A., \& Tifft, W. G. 1976 ApJ, 205, 696

Grevesse, N., \& Sauval, A. J. 1998, Space Sci. Rev. 85, 161

Haardt, F., \& Madau, P. 1996 ApJ, 461, 20

Haardt, F., \& Madau, P. 2001, in Clusters of Galaxies and the High-Redshift Universe Observed in X-Rays, ed. B. M. Neumann \& J. T. V. Tran.

Haynes, M. J., \& Giovanelli, R. 1985, AJ, 90, 2445

Hoyle, F., Rojas, R. R., Vogeley, M. S., \& Brinkman, J. 2005, ApJ, 620, 618

Kirshner, R. P., Oemler, A., Schechter, P. L., \& Shectman, S. A. 1981, ApJ, 246, L57

Madau, P., Ferrara, A., \& Rees, M. J. 2001, ApJ, 555, 92

McLin, K., M., Stocke, J. T., Weymann, R. J., Penton, S. V., \& Shull, J. M. 2002, ApJ, 574, L115

Moos, H. W., et al. 2000, ApJ, 538, L1

Morris, S. L., Weymann, R. J., Savage, B. D., \& Gilliland, R. L. 1991, ApJ, 377, L21

Penton, S. V., Stocke, J. T., \& Shull, J. M. 2000a, ApJS, 130, 121

Penton, S. V., Shull, J. M., \& Stocke, J. T. 2000b, ApJ, 544, 150

Penton, S. V., Stocke, J. T., \& Shull, J. M. 2002, ApJ, 565, 720

Penton, S. V., Stocke, J. T., \& Shull, J. M. 2004, ApJS, 152, 29

Pettini, M., Madau, P., Bolte, M., Prochaska, J. X., Ellison, S. L., \& Fan, X. 2003, ApJ, 594, 695

Prochaska, J. X., Weiner, B. J., Chen, H-W., \& Mulchaey, J. S. 2006, ApJ, 643, 680

Ricotti, M., Gnedin, N., \& Shull, J. M. 2002, ApJ, 575, 49
Ricotti, M., Gnedin, N., \& Shull, J. M. 2007, ApJ, to be submitted

Rojas, R. R., Vogeley, M. S., Hoyle, F., \& Brinkman, J. 2004, ApJ, 617, 50

- 2005, ApJ, 624, 571

Schaye, J. 2001 ApJ, 559, 507

Schaye, J., Aguirre, A., Kim, T.-S., Theuns, T., Rauch, M., \&

Sargent, W. L. W. 2003, ApJ, 596, 768

Shull, J. M., Roberts, D., Giroux, M. L., Penton, S. V., \& Fardal, M. A. 1999, AJ, 118, 1450

Simcoe, R. A., Sargent, W. L. W., \& Rauch, M. 2004, ApJ, 606, 92

Songaila, A. 2001, ApJ, 561, L153

Spergel, D. N., et al. 2007, ApJS, 170, 377

Stocke, J. T., Shull, J. M., \& Penton, S. V. 2006, in Planets to Cosmology: Essential Science in the Final Years of the Hubble Space Telescope, ed. M. Livio \& S. Casertano, (Cambridge: CUP), 111

Stocke, J. T., Penton, S. V., Danforth, C. W., Shull, J. M., Tumlinson, J., \& McLin, K. M. 2006, ApJ, 614, 217

Stocke, J. T., Shull, J. M., Penton, S. V., Donahue, M., \& Carilli, C. L. 1995, ApJ, 451, 24

Telfer, R. C., et al. 2002, ApJ, 565, 773

Tumlinson, J., Venkatesan, A., \& Shull, J. M. 2004, ApJ, 612, 602

Tumlinson, J., Shull, J. M., Giroux, M. L., \& Stocke, J. T. 2005, ApJ, 620, 95

Weymann, R. J., et al. 1998, ApJ, 506, 1 